\title{
The suffix effect: How many positions are involved?
}

\author{
RANDALL W. ENGLE \\ University of South Carolina, Columbia, South Carolina 29208
}

\begin{abstract}
Three experiments tested the effect of the availability of the last item on the recall performance for earlier list items in a stimulus suffix paradigm. A decrement due to the suffix occurred for preterminal positions even when the last item was made available. This preterminal suffix effect was found to be more extensive in a condition in which subjects were free to recall the end items first than in a condition in which subjects were forced to recall in a strict left-to-right fashion.
\end{abstract}

When a redundant speech sound is appended to the end of an auditorily presented list, recall performance is impaired for those items at the end of the list when compared with lists followed either by no sound or by a nonspeech sound such as a tone or buzz. This phenomenon is called the suffix effect, and it is important because of the argument by Crowder and Morton (1969) that the effect is a reflection of the contribution of the auditory sensory memory or echoic memory to recall in the nonsuffix control condition. In fact, inferences about echoic memory have been drawn almost exclusively from experiments using the suffix procedure (Crowder, 1972, 1975).

The suffix effect is most pronounced for the last serial position in the list, but it is frequently observed for the three or four terminal positions. Crowder (1978) has argued that the decrement in performance for only the last item should be considered important to theorizing about echoic memory. But, as he points out (see Crowder, 1978, Footnote 4), there are few data regarding this issue. If, indeed, the suffix effect is a reflection of the contribution of echoic memory to recall of auditorily presented lists, then how far the suffix effect extends into the list and whether the decrement for preterminal positions is also a reflection of echoic memory are nontrivial questions.

The purpose of this paper is to address this issue of the cause of suffix effect decrements for positions immediately prior to the last one. Two hypotheses come to mind as explanations for what I will call the preterminal suffix effect. One is that, indeed, the preterminal items also benefit from the lingering echoic trace and that the suffix acts as a mask for the echoic

This research was supported in part by Grant HD-11114 from the National Institute of Child Health and Human Development. I wish to thank Lydia Wilson Neely and David Stagg for help with data collection and analysis. Parts of Experiment 3 were suggested by an appropriately cautious anonymous reviewer. Requests for reprints should be sent to Randall Engle, Department of Psychology, University of South Carolina, Columbia, South Carolina 29208. information persisting for these items as well as for the terminal positions. This hypothesis does not view the preterminal suffix effect as qualitatively different from the effect of the suffix on recall of the last item. The difference would be one of degree, with the preterminal items simply having less of an echoic trace than the terminal item. This hypothesis has been viewed as objectionable because it does not fit well with estimates of the duration of the echoic memory. As Crowder (1978) points out, estimating the duration of the echoic trace has been a particularly sticky problem, but attempts using a delayed suffix suggest about 1-2 sec as a reasonable appraisal (Crowder, 1978). If this figure is accurate, then preterminal suffix effects should not be observed with presentation rates as slow as 2 or $4 \mathrm{sec} /$ item. Since such preterminal suffix effects are found with rates this slow (Watkins \& Watkins, in press), this suggests either that this hypothesis is wrong or that echoic information persists for longer than the 1- to 2-sec estimate.

An alternative hypothesis is that in the nonsuffix condition, the last item is the only one for which there is an echoic trace available to aid recall. The recall of this last item might serve as a retrieval cue for recall of other items, particularly those items immediately prior to the terminal one. It would be assumed that the suffix masks echoic information for only the last item, but with this item no longer available, it could not serve as a retrieval cue for the preterminal items. This view, which I will call the lost retrieval cue hypothesis, predicts that, if we resupply the last item, the preterminal suffix effects should disappear. This is the prediction tested in the three experiments reported here.

\section{EXPERIMENT 1}

This experiment was prompted by the prediction of the lost retrieval cue hypothesis that providing the subject with the last item prior to recall would eliminate preterminal suffix effects.

\section{Method}

Subjects. Thirty students from elementary psychology 
courses volunteered for course credit. They were tested in groups of two to five per group in a small classroom.

Materials and Procedure. The lists were nine items in length and were generated from a random number table with the constraint that no digit could appear twice in a list. The lists were presented to the subject in three blocks of 18 lists each, and the position of digits within lists was counterbalanced so that, within a block of 18 lists, each digit appeared twice in each serial position. The lists were recorded by a female voice at $.8 \mathrm{sec} /$ digit. The three blocks of lists each represented a different condition in the experiment. The nonsuffix condition (NS) was the control condition and simply had a $500-\mathrm{msec}$ tone occur in synchrony following the last item. The suffix condition (S) had the word "Go" follow the last digit, again, in synchrony. The condition of most interest had the suffix "Go" follow the last digit, but in this case, the suffix was immediately followed by the experimenter's holding up a white card with the last digit printed on it in bold black ink. This condition, S-VLD, was predicted by the lost retrieval cue hypothesis to eliminate the effect of the suffix on preterminal items. The order of conditions was counterbalanced so that each group of five subjects received one of the six possible orders of conditions.

The subjects were told what to expect at the beginning of each session and immediately before each condition was presented. For example, prior to the S-VLD lists, the subjects were told that they would hear nine digits and then the word "Go" and that the last digit they heard would be held before them immediately afterward. They were told it would be visible until the next list was ready to begin and that they should always get this number correct. Recall was written on answer sheets with nine boxes per list, and instructions emphasized that the digits should be written in a left-to-right manner with no regressions.

\section{Results}

The recall performance was analyzed using two different scoring procedures. The all-or-none procedure is the one typically used in suffix effect studies and allowed credit if and only if the correct digit was recalled in the correct serial position. The partial credit procedure was an attempt to give credit for items recalled, but recalled out of position. This procedure allowed one point to the serial position if the correct digit was recalled in the correct position. If the item from a given input position was recalled one position away from the correct one, the correct position was credited with .75 ; if the digit was recalled two positions away, the correct position was credited with .5 ; three positions away, with .25; and four or more positions away, with 0 points. The two analyses yielded slightly different $F$ values, but the conclusions were identical. For this reason, and because it is typical of suffix effect studies, the discussion below will be of the analysis using the all-or-none scoring.

The mean proportion correct at each serial position for the three conditions is shown in Figure 1. While the analysis yielded significant main effects of conditions $[\mathrm{F}(2,48)=20.0, \mathrm{p}<.01, \mathrm{MSe}=.046]$ and Serial Position $[F(8,192)=57.2, p<.01, \mathrm{MSe}=.046]$, the finding of most interest was the Conditions by Serial Position interaction $[F(16,384)=9.4, p<.01, \quad \mathrm{MSe}=.011]$. Differences between conditions at each serial position were analyzed by Tukey HSD tests at the .05 level. These tests revealed significant decrements in perfor-

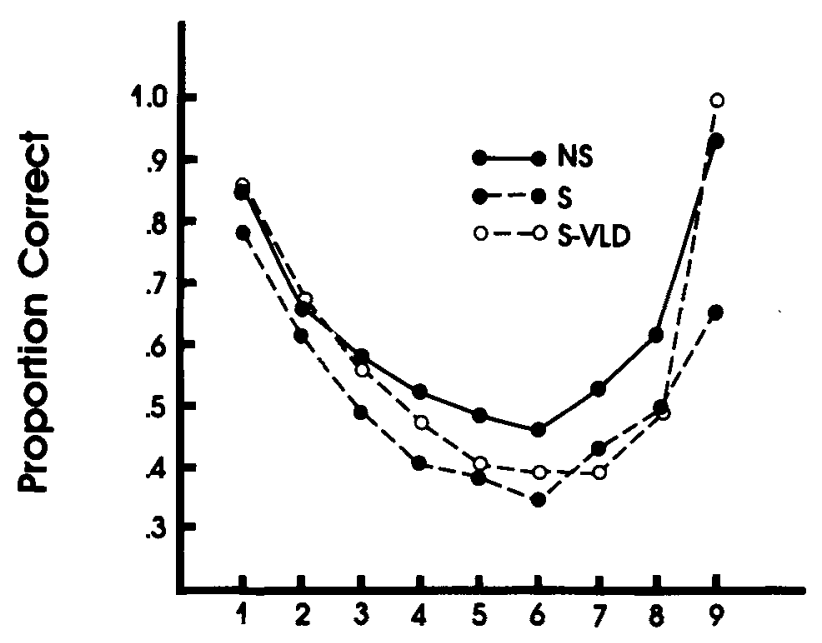

\section{Serial Position}

Figure 1. Proportion correct recall as a function of input position for the nonsuffix condition (NS), suffix condition (S), and suffix condition with redundant visual presentation of the last digit (S-VLD).

mance for the standard suffix condition at every serial position but the first two; in other words, there was a significant suffix effect for the final seven positions. The suffix condition followed by visual presentation of the last digit (S-VLD) resulted in significant decrements for Positions 5-8 when compared with the nonsuffix condition.

Obviously, the S.VLD condition did not eliminate the suffix effect for preterminal items, as had been predicted by the lost retrieval cue hypothesis. The suffix effect for Positions 5-8 was just as marked for this condition as for the standard suffix condition.

It is possible that the decrement in performance for the preterminal items in the S-VLD condition resulted from a distraction of the subjects, such as shifting their attention from listening to the digits to looking at the visual presentation of the last digit. To rule out this hypothesis, some method was needed that would constitute a suffix procedure and keep the last digit available as a retrieval cue, but which would not require a shifting of attention from one modality to another. One procedure that seemed to satisfy these criteria was to simply repeat the last digit as the suffix.

Rate of presentation was also varied in the next experiment to determine whether the preterminal suffix effects observed in Experiment 1 generalize to faster rates of presentation. This is a particularly important manipulation in terms of Crowder and Morton's (1969) theory of echoic memory. If the echoic trace is time dependent, as well as prone to interference from subsequent speech masks, the extent of the suffix effect over the end of the list should be tied to the rate of presentation for the items. Quickly presented lists should show the suffix effect over more preterminal 
positions than should slowly presented lists because, for an item in a given input position, less time passes between the presentation of that item and the cue for recall with fast presentation than for an equivalent item with slow presentation. If the lost retrieval cue explanation is valid, then making the last item available either visually or by making the suffix be a repetition of the last item should eliminate the preterminal suffix effect for both fast and slow rates of presentation.

\section{EXPERIMENT 2}

\section{Method}

Subjects. Thirty-four students of elementary psychology volunteered to be tested for course credit. They were tested individually in a small sound-shielded room.

Procedure. Eighty lists of nine digits each were randomly generated by a program, with the constraint that no digit be repeated in a list. The lists were divided into four blocks of 20 lists each, with each block to be presented in one of the four conditions. Presentation rate was $1 \mathrm{digit} / \mathrm{sec}$ for half of the subjects and 4 digits/sec for the other half of the subjects. The four conditions, three of which were identical to the first experiments, were: (1) the nonsuffix control condition (NS), (2) the standard suffix condition (S), in which the suffix "Go" was appended at the end of the list in synchrony with the list items, (3) a suffix condition in which the "Go" was followed by visual presentation of the last digit (S-VLD), and (4) a suffix condition in which the last digit was always repeated as the suffix (S-LDR). Conditions was a within-subjects variable, and the order of conditions was randomized for each subject. The instructions were identical to those used in Experiment 1 with the necessary additions for the S-LDR condition, emphasizing that the last digit had to be recalled only once and that the repetition could be ignored or used as a recall cue, whichever was desired. Recall was written on answer sheets with nine boxes drawn on them for each list.

\section{Results}

The all-or-none scoring procedure was used for this experiment, and the results are shown in Figure 2, with the 1-digit/sec group on the left and the 4-digit/sec group on the right. Obviously, the preterminal suffix effect remains regardless of whether the last item is made available by visual presentation, S-VLD, or by the last item's being repeated as the suffix, S-LDR. This was supported by the conditions main effect $[F(3,96)=22.4$, $\mathrm{p}<.01, \mathrm{MSe}=.03]$ and the Condition by Serial Position interaction $[F(24,768)=22.6, p<.01, \mathrm{MSe}=.01]$.

Post hoc analyses revealed significant preterminal suffix effects for all three suffix conditions for Position 8 for the 1-digit/sec rate and for Positions 7 and 8 for the 4-digit/sec rate. The S-VLD condition replicated the findings of the first experiment in finding the preterminal suffix effect. The S-LDR condition, likewise, showed the preterminal suffix effects even when the last digit was made available without shifting modalities. These findings appear to belie the validity of the lost retrieval cue hypothesis, and, consequently, they support the hypothesis that the preterminal suffix effects are a result of interference or masking of echoic information for these positions in much the fashion, if to less a degree, as occurs for the last item in the list.

Since Crowder and Morton's (1969) theory seems to make specific predictions about the effect of presentation rate, the two typical conditions, NS and S, were

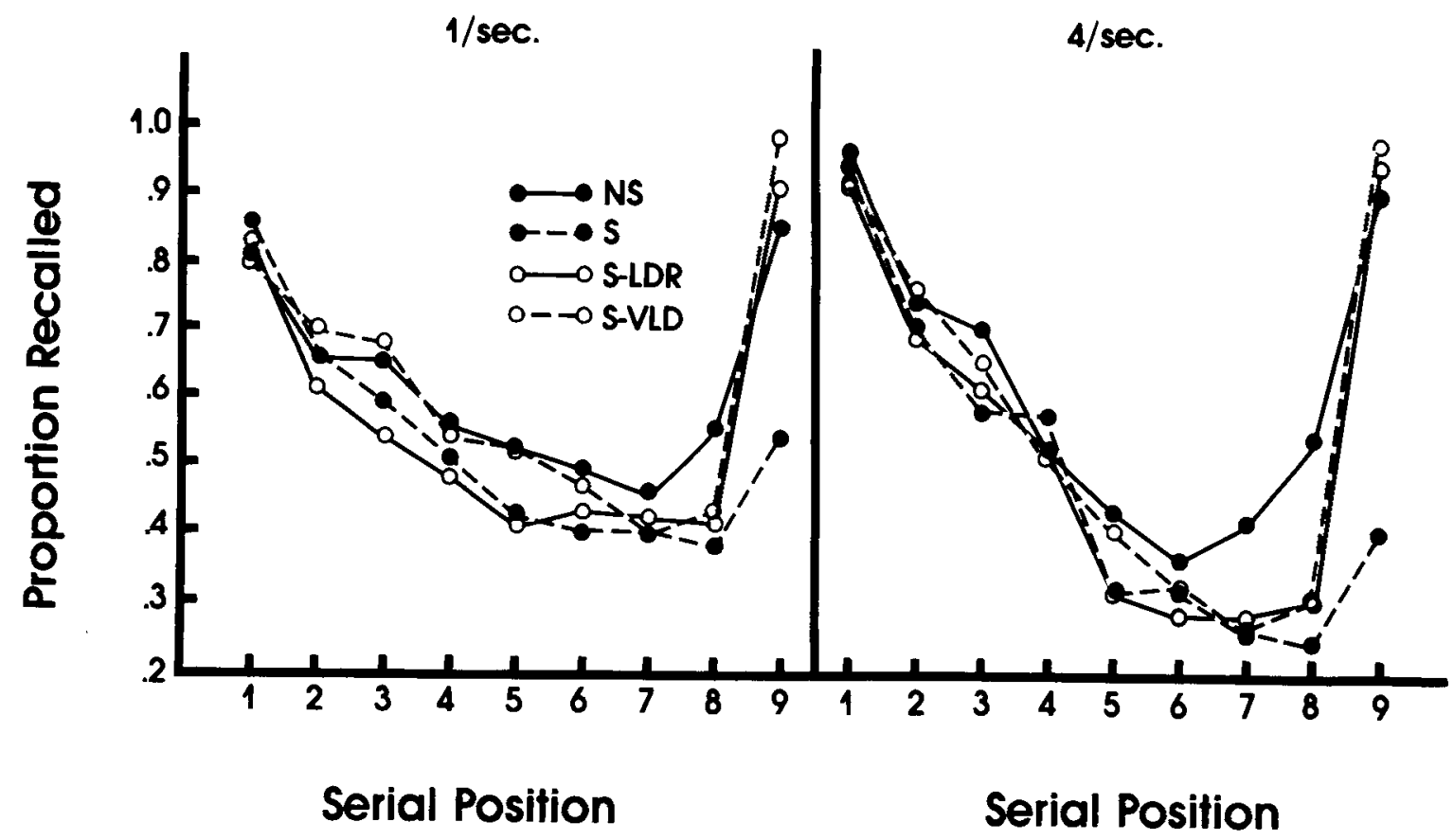

Figure 2. Proportion correct recall as a function of input position and rate of presentation for the nonsuffix condition (NS), suffix condition (S), and suffix conditions with visual last digit (S-VLD) and with last digit repeated (S-LDR). 
subjected to a separate analysis of variance for the effects of rate, conditions, and serial position. These data are redrawn in Figure 3 so that an easier comparison can be made.

Recall performance was about the same for the two rates, as evidenced by the absence of a rate main effect $(F<1.0)$. Also of interest was the fact that the magnitude of the suffix effect was slightly, but nonsignificantly, different for the two rates. The suffix caused a decrement of $13 \%$ at the 1-digit/sec rate and $22 \%$ at the 4-digit/sec rate, but the Rate by Condition interaction only approached significance $[F(1,32)=2.84$, $\mathrm{p}<.10$ ] . If only the last three positions are considered, however, the magnitude of the decrement caused by the suffix varied considerably with rate, with a $28 \%$ decrement occurring in the 1-digit/sec rate and a $51 \%$ decrement occurring in the 4-digit/sec rate. Inspection of Figure 3 shows that, while the magnitude of the suffix effect over all positions was marginally larger for the faster rate, the effect appears to have been much more concentrated over the final three positions, whereas for the slower rate, the decrement that did occur was spread over more positions. This is indicated by the significant Conditions by Rate by Serial Position interaction $[\mathrm{F}(8,256)=3.1, \mathrm{p}<.01, \mathrm{MSe}=.01]$.

An interesting note is that there is a general shift in the curves as we vary presentation rate. This is most clearly reflected by the nonsuffix curves, with the 4-digit/sec group showing better performance over the early positions and the 1-digit/sec group showing better performance over the middle and end positions. These conclusions are based on the significant Rate by Serial Position interaction $[F(8,256)=4.7, p<.01$, MSe $=.02]$, in combination with the absence of an overall main effect of rate, with mean proportion of .57 for

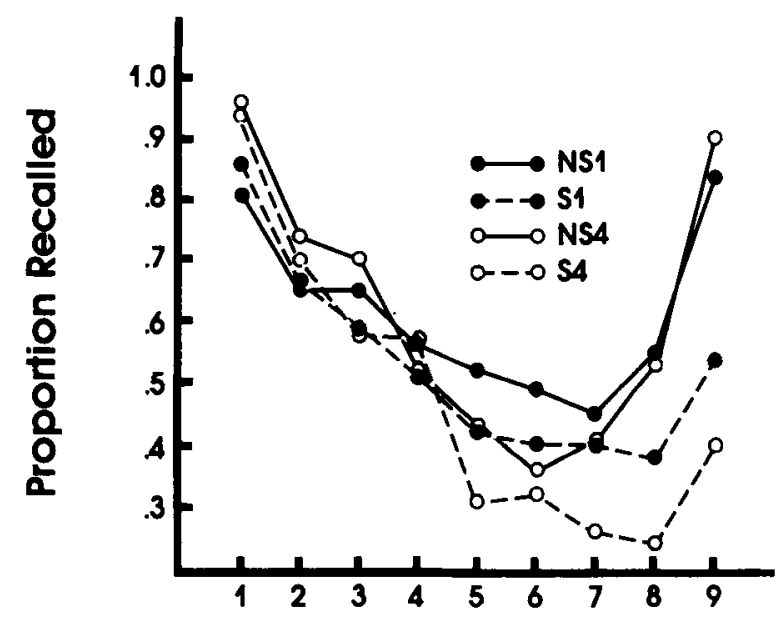

\section{Serial Position}

Figure 3. Proportion correct recall as a function of input position for the suffix and nonsuffix conditions for the 1-digit/ sec and 4-digit/sec rates of presentation. the 1-digit/sec group and .55 for the 4-digit/sec group.

A rather obvious defect in the first two experiments is that the different suffix conditions were blocked so that the subject knew when a list was being presented what the suffix would be like. It is possible that this led to the subject's attending or rehearsing the lists differently in the various conditions. The third experiment was designed to remedy this defect.

Another potential problem with the first two experiments was that the suffix in the straight suffix condition ("Go") was always the same item, whereas the suffix in the last-digit-repeated condition and the visual-lastdigit condition varied from list to list. The next experiment controlled for this by using, as one type of suffix, a letter selected randomly from a pool the same size as that from which the last digit was chosen.

In addition, the third experiment was designed to look at the interaction of type of recall instructions with suffix conditions. It might have been noted that the suffix effect extended over more positions in Experiment 1 than in Experiment 2. The two experiments differed in many ways, but the nature of the recall instructions seemed to be a variable that might affect the results in the manner just described.

It is typical in our laboratory to instruct subjects in suffix effect studies to recall in a serial manner, but subjects are typically not closely monitored to insure that recall is performed as requested. In reviewing the procedures for the first two experiments, we noticed that it appeared that, as a consequence of having two different experimenters, there was more emphasis given to the left-to-right nature of recall in Experiment 2 than in Experiment 1. To determine whether this would influence the extent of the suffix effect, nature of recall instructions was manipulated in the third experiment

\section{EXPERIMENT 3}

\section{Method}

Subjects. Forty eight students from the same population used in the first two studies were tested in small groups.

Procedure. Seventy-two digit lists of nine items each were generated by a program, with the constraints that no digit be repeated in a list and, over lists, each digit occur in each serial position an equal number of times. The lists were composed of the digits 16 and $8-10$. The lists were recorded at a 2 -digit/sec rate and were presented over stereo speakers in a small soundshielded room to small groups of subjects.

The only between-subjects variable was the nature of recall instructions. Twenty-seven of the subjects received strict serial recall instructions. They were told to recall the lists in exactly the same manner in which they were presented, writing a digit in the leftmost box on the recall sheet before going to the next one. It was further emphasized that this mode of recall was probably unlike what they would want to do, but that it was imperative that they recall in exactly the manner described. If they were unsure about the correct digit to be written in a particular box, they were to guess. The recall behavior of these subjects was closely monitored, and the data of three subjects were eliminated due to failure to follow instructions.

The other 21 subjects, in the free condition, were instructed 
to recall the digits in any sequence they wished as long as the correct digit was in the box representing the correct input position. They were also instructed to guess if unsure about which digits should go in which boxes.

The other variables, all within subjects, were suffix condition and serial position. There were four different suffixes, one of which followed each list. The order of suffix condition was random across the 72 lists, so that the subject could not anticipate which suffix would occur. The four suffixes were: (1) a tone (T), (2) the last digit repeated (LDR), (3) a letter (L) chosen from a pool of nine letters in a manner analogous to the way the last digit was chosen, and (4) the word "Go."

\section{Results}

Scoring was performed using both the all-or-none procedure and the partial procedure described for Experiment 1 . The two procedures again yielded identical conclusions, and, again, only the results of the all-or-none procedure will be presented.

It is clear from the results, shown in Figure 4, that

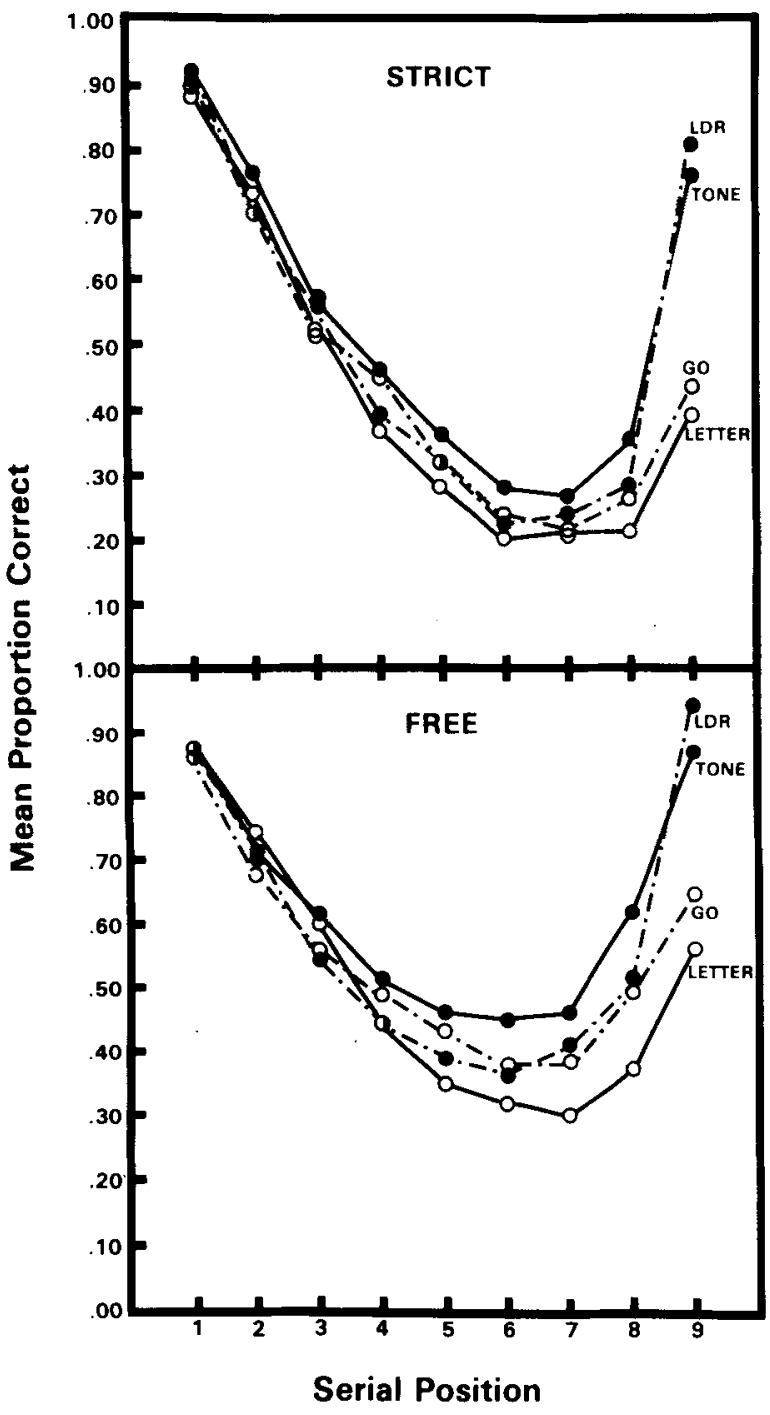

Figure 4. Proportion correct recall as a function of input position, suffix condition, and nature of recall instructions. the preterminal suffix effect is still observed even if the subject cannot predict which suffix will occur, but it is also clear that the extent of the suffix effect depends on recall instructions. While the suffix caused a decrement for Positions 4-9 in the free recall condition, the decrement occurred only for Positions 8 and 9 in the strict recall condition. This was supported by the significant Recall Instructions by Suffix Condition by Serial Position interaction $[F(24,1104)=1.7, p<.05, \mathrm{MSe}=.009]$.

The main effect of recall instructions $[F(1,46)=5.5$ $p<.025, \mathrm{MSe}=.56]$ and the Instructions by Serial Position interaction $[\mathrm{F}(8,368)=7.8, \mathrm{p}<.001$, MSe $=$ .04] reflected the fact that subjects in the free condition performed about $17 \%$ better than those in the strict condition, and nearly all of this difference was over the final five serial positions. The facts that these were the same positions showing significant preterminal suffix effects for the free group and that the curve for the poorest suffix condition in the free recall group (L) was nearly identical to the nonsuffix condition (T) in the strict recall group may be coincidental. On the other hand, it may suggest that the free recall group had available to them at the initiation of recall a source of information about the terminal items that was highly transient and was lost both in the process of a strict left-to-right recall and as a consequence of a speech suffix. This additional source of information could be sensory in nature, such as the echoic trace or something more refined. Which explanation is correct remains to be determined.

\section{GENERAL DISCUSSION}

These three experiments were directed to the hypothesis that the decrement in recall in the suffix condition for positions prior to the last one occurs because the last item is no longer available to serve as a retrieval cue to the items presented just prior to it. All three experiments showed marked decrements in recall for preterminal positions, even when the last item was made available either by visual presentation of the last digit following the suffix or by repetition of the last digit as the suffix. Further, it makes no difference whether the subject can or cannot predict the particular suffix. This appears to disconfirm the lost retrieval cue hypothesis.

One alternative hypothesis for the preterminal suffix effect is that the echoic trace persists much longer than has previously been believed and that more list items than just the last one benefit from auditory presentation by being stored in the echoic memory in addition to being stored in the modality independent of short-term memory. This hypothesis is less objectionable in light of recent arguments by Watkins and Watkins (1979) that the echoic trace persists for considerably longer than the $1-2 \mathrm{sec}$ estimated by Crowder (1978), perhaps $15 \mathrm{sec}$ or longer.

Another possible explanation is that the suffix 
causes the decrement in performance on the preterminal positions and on the last position through different mechanisms. Morton (1976) has suggested that, in addition to the passive overwriting of echoic information, the suffix intrudes upon the attention of the subject, who cannot distinguish the suffix from list items. This automatic grouping notion, first proposed by Kahneman (1973) to explain the entire suffix effect, might be used to explain the preterminal suffix effect. It is possible that the suffix effect for the final position is a result of the masking of echoic information by the suffix and that the decrement for preterminal positions is caused by attentional factors not related to the storage of primitive auditory information. However, this theory would predict that any condition increasing the distinctiveness of the suffix and list items would lessen the decrement caused for preterminal positions but not for the final position. In fact, Morton (1976) showed that three identical suffixes in sequence, a condition that supposedly caused the list items and suffixes to be organized into separate groups or chunks, reduced the suffix effect for the final position but, if anything, increased it for the preterminal positions. This suggests that the automatic grouping explanation is not correct either, but it does suggest that the preterminal and terminal suffix effects are mediated by different mechanisms.

Baddeley and Hull (1979) have recently argued that the suffix effect for preterminal positions is mediated by a process different from the one that mediates the decrement for the last position. They base their argument on a set of studies that showed that, as the number of syllables in the suffix increased, the preterminal suffix effect also increased. It is difficult to tell from their results section what effect this manipulation had on the last item, because the effect on the last item was figured with respect to the performance on the previous item in the same condition and not with respect to the last position in the control condition. It appears that if the last position in the control condition is taken as the reference, then the increase in syllables in the suffix has no effect on the size of suffix effect on the last position. In this case, their studies show that increasing the number of syllables in the suffix increases the preterminal suffix effect beyond what it would normally be but has no noticeable effect on the terminal suffix effect. This does not necessarily mean that the suffix is not at least contributing to the preterminal suffix effect by interfering with echoic information for those items, but it is rather suggestive of a two-factor theory of the two suffix effects.

Baddeley and Hull (1979) argue that the preterminal suffix effect and the response prefix effect are caused by a common mechanism. They assume the common mechanism is a delay in rehearsal caused by the time it takes to say the prefix or process the suffix. It is not clear, however, why the subject would process the redundant suffix or even how the subject could process a nonsensical speech sound (e.g., a word played in reverse through the tape player) in a manner that would interfere with the rehearsal buffer. While the precise nature of a separate mechanism for the preterminal suffix effect is unclear, there appears to be some support for its existence. One further line of support comes from our laboratory: Balota and Engle (Note 1) have found that extensive practice with suffix lists reduces the magnitude of the preterminal suffix effect but not the effect on the last position. Even if the two-factor theory is not correct and the preterminal suffix effect is a result of masking of lingering primitive auditory information, it is not entirely clear what this means to the theory of echoic memory. It undoubtedly means that echoic memory persists longer than has previously been assumed, but whether any theory explaining the suffix effect for the single final position can, unaltered, also explain suffix effects for preterminal positions as well remains to be seen. For example, Crowder (1978) explains the diminution of the suffix effect with multiple suffixes by arguing that the additional suffixes partly mask the effect of the original suffix through a form of lateral inhibition. This would seem to suggest, however, that the effect of a single suffix would be to inhibit the masking effect of the last digit on the echoic trace for the penultimate digit, thus increasing its probability of recall compared with the control condition. This obviously is not the case and points out the necessity for consideration of the preterminal suffix effect in any theory of echoic memory.

\section{REFERENCE NOTE}

1. Balota, D. A., \& Engle, R. W. Structural and control elements in the stimulus suffix effect. Paper presented at the meeting of the Midwestern Psychological Association, St. Louis, Missouri, 1980 .

\section{REFERENCES}

Baddeley, A., \& Hull, A. Prefix and suffix effects: Do they have a common basis? Journal of Verbal Learning and Verbal Behavior, 1979, 18, 129-140.

Crowder, R. G. Visual and auditory memory. In J. F. Kavanaugh \& I. G. Mattingly (Eds.), Language by eye and by ear: The relation between speech and learning to read. Cambridge, Mass: M.I.T. Press, 1972.

Crowder, R. G. Inferential problems in echoic memory. In P. M. A. Rabbit \& S. Dornic (Eds.), Attention and performance $V$. New York: Academic Press, 1975.

Crowder, R. G. Mechanisms of auditory backward masking in the stimulus suffix effect. Psychological Review, 1978, 85, 502-524.

Crowder, R. G., \& Monton, J. Precategorical acoustic storage (PAS). Perception \& Psychophysics, 1969, 5, 365-373.

Kahneman, D. Attention and effort. Englewood Cliffs, N.J: Prentice-Hall, 1973.

Morton, J. Two mechanisms in the stimulus suffix effect. Memory \& Cognition, 1976, 4, 144-149.

Watkins, O. C., \& Watkins, M. J. The modality effect and echoic persistence. Journal of Experimental Psychology: General, in press.

(Received for publication June 5, 1979; revision accepted January 7, 1980.) 